\title{
Clinical characteristics and outcome of pauci-immune glomerulonephritis in African Americans
}

Duvuru Geetha, MD, MRCP (UK) $)^{a,{ }^{*}}$, Caroline J. Poulton, MSW ${ }^{b}$, Yichun Hu, MS ${ }^{b}$, Philip Seo, MD ${ }^{\mathrm{a}}$, Julie Anne G. McGregor, MD ${ }^{\mathrm{b}}$, Ronald J. Falk, MD ${ }^{\mathrm{b}}$, and Susan L. Hogan, PhD, MPH

aDepartment of Medicine, Johns Hopkins University School of Medicine, Baltimore, MD

bDepartment of Medicine, University of North Carolina, Chapel Hill, NC

\begin{abstract}
Objectives-Pauci-immune glomerulonephritis is rare in African Americans (AA) and the clinical presentation and treatment outcomes of vasculitis have not been well described.

Methods-We identified patients who were 2-92 years of age between 1983 and 2011 with a diagnosis of biopsy-proven pauci-immune glomerulonephritis (GN) at any point during their disease course. Comparing AA to Caucasian patients, we examined demographics, clinical features at presentation, treatment and outcomes of relapse, end-stage renal disease (ESRD), and death.
\end{abstract}

Results-Of the 672 patients, 75 were AA with the remainder being Caucasian. Compared to Caucasians, disease onset in AA was at an earlier age (52 vs. 57 years, $p=0.05$ ) and was more often myeloperoxidase (MPO)-anti-neutrophil cytoplasmic antibody (ANCA) positive (71\% vs. $54 \%, p=0.01$ ). AA patients had a shorter median time between onset of symptoms and biopsy compared to Caucasians [median (IQR): $0.23(0.00,1.22)$ months vs. $0.66(0.00,3.62)$ months, $p=$ 0.003]. Median [Interquartile range (IQR)] follow-up in months was $28(5,52)$ in AA and $26(10$,

$55)$ in Caucasian patients. Median estimated glomerular filtration rate was similar at presentation ( 21 vs. $22 \mathrm{ml} / \mathrm{min} / \mathrm{m}^{2}$ ). Both groups had similar induction treatment regimens. There was less favorable treatment response among AA compared to Caucasians for initial treatment resistance $(28 \%$ vs. $18 \%, p=0.05)$ and complete remission $(72 \%$ vs. $82 \%, p=0.05)$. There were no differences in the number of renal relapses or number of deaths between the 2groups. Overall, in multivariable analyses controlling for age, race, ANCA type, and entry serum creatinine, there were not differences by race in treatment response, renal relapse, ESRD, or death over the entire time of follow-up. Conclusions: AA patients with pauci-immune GN are younger and more often MPO-ANCA positive compared to Caucasians. Despite a shorter time to diagnosis for AA patients, there were no differences compared to Caucasians in treatment response, ESRD, renal relapse, or death rates by race over the entire duration of follow-up.

\section{Keywords}

Pauci-immune glomerulonephritis; ANCA vasculitis; African Americans; Treatment outcome

(c) 2013 Elsevier Inc. All rights reserved.

"Correspondence to: Duvuru Geetha, MD, MRCP (UK), Division of Nephrology, Johns Hopkins Bayview Medical Center, Johns Hopkins University, 301 Mason Lord Drive, Baltimore, MD 21224. gduvura@jhmi.edu (D. Geetha). 


\section{Background}

Pauci-immune crescentic glomerulonephritis (GN) accounts for $80 \%$ of cases of rapidly progressive glomerulonephritis [1-3]. The majority of cases of pauci-immune GN can be attributed to primary systemic small vessel vasculitides including granulomatosis with polyangiitis (GPA), microscopic polyangiitis (MPA), and eosinophilic granulomatosis with polyangiitis (eGPA). Some cases involve only the kidneys and can be categorized as renallimited vasculitis (RLV) [4]. Anti-neutrophil cytoplasmic antibody (ANCA) is a serologic marker for pauci-immune glomerulonephritis. ANCA is present in $80-90 \%$ of patients with pauci-immune GN and therefore these vasculitides are also called ANCA-associated vasculitis (AAV) [5]. AAV occurs in older adults with no clear gender predilection and is rare among African Americans (AA) [6]. Environmental factors and genetic susceptibility are involved in inducing ANCA production. Recently, the HLA-DRB1*15 allele was found to be associated with a higher risk of AAV in AA patients with proteinase 3 (PR3)-ANCA compared to Caucasians [6]. There is evidence to suggest differences in disease expression and outcomes among AA with other autoimmune diseases like lupus, rheumatoid arthritis, and scleroderma [7].

This study aims to examine demographics, clinical features at presentation, and treatment, and to correlate these variables with treatment response, rates of end-stage renal disease (ESRD), time to first disease relapse, and death in AA patients compared to Caucasian patients with biopsy-proven pauci-immune crescentic GN. The identification of race-related differences in disease characteristics and outcomes may provide clues to the etiology and pathogenesis of AAV.

\section{Material and methods}

\section{Study population}

The study population combined patients from a retrospective cohort from Johns Hopkins Hospital (Maryland) and an inception cohort from the University of North Carolina Kidney Center's Glomerular Disease Collaborative Network (GDCN), which has previously been described [8]. The study included patients with histologic confirmation of pauci-immune glomerulonephritis by a kidney biopsy at any time point during the course of their vasculitis from 1983 to 2011. "Pauci-immune" was defined as "the intensity of glomerular immunoglobulin staining by direct immunofluorescence assay in renal sections being 0 to $1+$, from a staining scale of 0 to 4+". Patients were classified as GPA, MPA, or eGPA as defined by the Chapel Hill Consensus Conference [9] and as RLV if they had pauci-immune necrotizing and/or crescentic glomerulonephritis without any overt signs of systemic vasculitis. The patients were followed up until ESRD, transplant, death, transfer to another facility, or until the end of the follow-up period (December 31, 2011). An exclusion criterion for this study was race besides AA or Caucasian. The study protocol was approved by the Institutional Review Board at each institution. 


\section{Data acquisition}

Clinical data-Data regarding patient demographics at the time of diagnosis, new vs. established diagnosis, clinical features at presentation, occurrence of disease relapse, need for renal replacement therapy at the time of diagnosis and at last follow-up, and details of induction and maintenance immunosuppression were collected from review of medical records. Kidney involvement was defined by a diagnostic renal biopsy or with the presence of active urine sediment with or without renal insufficiency. Remission was defined as stabilization or improvement in serum creatinine, resolution of hematuria, and absence of extra-renal signs of vasculitis for 1 month [8]. Treatment resistance was defined as progressive deterioration of renal function with the persistence of active urine sediment or development of new or persistent signs of extra-renal vasculitis despite treatment with immunosuppressive therapy for at least 1 month [8]. Relapse was defined as occurrence of signs and symptoms of vasculitis in any organ requiring a change in immunosuppressive therapy after achieving remission [6].

Laboratory data-Peak serum creatinine level at the time of diagnosis was recorded. Renal function was measured using the 4-variable modification of diet in renal disease (MDRD) formula [10]. ANCA testing was done by standard indirect immunofluorescence assay on ethanol-fixed neutrophils for cytoplasmic ANCA (c-ANCA) and perinuclear ANCA (p-ANCA). PR3 and myeloperoxidase (MPO) testing was done by direct enzymelinked immunosorbent assay (ELISA) with commercially available kits. An ANCA titer of less than 10 was considered to be negative for c-ANCA and p-ANCA (reference range: 010). PR3 and MPO levels of less than 20 units were considered negative (reference range: 0-20). If the testing for c-ANCA, p-ANCA, PR3 ELISA, and MPO ELISA was negative, patients were labeled as having ANCA-negative vasculitis. Patients were categorized as having cytoplasmic and/or proteinase 3-ANCA (thereafter referred to as PR3-ANCA) or perinuclear and/or myeloperoxidase-ANCA (thereafter referred to as MPO-ANCA). pANCA alone required negative anti-nuclear antibody test for the GDCN cohort but this was not required for the Hopkins cohort.

\section{Outcomes}

The principal outcomes of interest in this cohort were complete remission, initial treatment resistance, renal recovery after acute dialysis at diagnosis, time to first renal relapse, ESRD (early or late), and death. ESRD was defined as the need for ongoing renal replacement therapy in the form of dialysis or renal transplantation. Early ESRD was defined as the need for renal replacement therapy within the first 3 months of diagnosis of pauci-immune GN, and late ESRD was defined as the need for renal replacement therapy after the first 3 months of diagnosis of pauci-immune GN.

\section{Statistical analyses}

All analyses were stratified based on race (AA vs. Caucasian) as determined from medical records, with all other races excluded. Fisher's exact test was used to evaluate differences by race for categorical variables. Wilcoxon rank sum test was used to compare continuous distributions between racial groups. Median and interquartile ranges (IQR) were reported for 
continuous measures with skewed distributions. For initial analysis of outcomes of interest, we used a two-sample $t$ test to compare AA and Caucasians. Multivariable analysis for treatment resistance was done using logistic regression. Cox proportional hazards models were evaluated to obtain multivariate hazards ratios and $95 \%$ confidence intervals for the outcomes of relapse, ESRD, and death over the entire duration of follow-up. Evaluation for relapse was limited to those who went into remission as a result of their initial treatment. We controlled for the following predictor variables in multivariate models, with the goal of understanding differences in race independent of these measures: age, sex, and ANCA type. A $p$-value of 0.05 or less was considered to be significant. All analyses were done using SAS software (version 9.3, SAS Institute, Cary, NC).

\section{Results}

Overall, 708 patients with biopsy-proven pauci-immune GN were identified (613 from the GDCN and 95 from Johns Hopkins). Thirty-six patients with race other than African American or Caucasian were excluded. Among the 672 with race identified, 75 (11\%) were AA with the remainder being Caucasian. Table 1 lists the demographics and baseline characteristics by race. AA patients were younger at onset of disease with a mean age of 52 years compared to Caucasians with a mean age of 57 years $(p=0.05)$. ANCA negativity was similar between AA and Caucasians (4\% and 2\%, respectively), and AA were more often MPO-ANCA positive compared to Caucasians ( $71 \%$ vs. $54 \%, p=0.01)$.

AA patients had a shorter median time between onset of symptoms and biopsy compared to Caucasians [median (IQR): $0.23(0.00,1.22)$ months vs. $0.66(0.00,3.62)$ months, $p=$ 0.003]. The frequencies of other organ system involvement were similar between the 2 racial groups. Baseline renal function was similar between groups, with median estimated glomerular filtration rate (eGFR) of 22 (IQR: 14, 45) $\mathrm{ml} / \mathrm{min} / \mathrm{m}^{2}$ in AA vs. 21 (IQR: 12, 38) $\mathrm{ml} / \mathrm{min} / \mathrm{m}^{2}$ in Caucasians. The duration of follow-up was also similar in both groups, with a median of 28 (IQR: 5, 52) months in AA and 26 (IQR: 10, 55) months in Caucasian patients.

The majority of patients in both groups were treated with the standard induction immunosuppressive therapy of cyclophosphamide and oral corticosteroids (Table 1). A minority of patients did not receive any immunosuppressive therapy for unclear reasons, and $4 \%$ of AA and $7 \%$ of Caucasians received other immunosuppressive therapy at induction including mycophenolate mofetil, azathioprine, methotrexate, rituximab, and intravenous immunoglobulin. There were no differences in the use of immunosuppressive therapy between AA and Caucasian patients (Table 1). However, AA patients were more likely to receive plasmapheresis compared to Caucasians ( $27 \%$ vs. $13 \%, p=0.005)$.

Initial treatment resistance was more common among AA than Caucasians (28\% vs. $18 \%$, respectively, $p=0.05$; Table 2). However, controlling for differences in peak serum creatinine at entry, age, sex, and ANCA specificity, there were no significant differences in treatment resistance between the groups $[\mathrm{OR}=1.49, \mathrm{CI}=(0.65,3.47), p=0.35]$ (Table 3 ).

Although median eGFR at presentation was similar by race as described above, AA patients required acute hemodialysis more often at the time of presentation, as compared to 
Caucasian patients (19\% vs. $13 \%, p=0.21)$. Among the 14 AA patients who required dialysis at presentation, $8(60 \%)$ recovered renal function; among 78 Caucasian patients requiring dialysis at presentation, $60(77 \%)$ recovered function $(p=0.18)$. AA patients were more likely to reach ESRD in the first 3 months ( $8 \%$ vs. $3 \%, p=0.04)$ in univariate analysis, but this association disappeared in multivariable analysis controlling for age, gender, serum creatinine at biopsy, and ANCA specificity $[\mathrm{HR}=2.46, \mathrm{CI}=(0.87,6.92), p=0.09]$. At last follow-up, 12 (16\%) AA patients and 53 (9\%) Caucasian patients had reached ESRD $(p=$ 0.05; Fig.), but in multivariable analysis controlling for age, gender, MPO vs. PR3-ANCA, and peak serum creatinine at entry, there was no difference by race, and age was the only significant factor predictive of overall ESRD (Table 3).

Among the 50 AA who achieved remission, 18 (36\%) experienced a disease relapse compared to 227 (49\%) of 464 Caucasians who experienced a disease relapse after achieving remission. Renal relapse occurred in 12 AA patients (24\%) compared to 137 (30\%) Caucasians $(p=0.51)$. There were no differences in time to disease relapse or renal relapse by race ( $p=0.89$ and 0.85 , respectively). There were $14(19 \%)$ deaths among AA and $144(24 \%)$ deaths among Caucasians during the follow-up period $(p=0.39)$. There were no differences by race in multivariable models for time to relapse or death over the entire follow-up controlling for age, gender, and ANCA specificity.

\section{Discussion}

This is the first descriptive study of an AA cohort of pauci-immune crescentic GN patients describing the demographics, clinical features at presentation, and treatment response in comparison to Caucasian patients with pauci-immune GN. Overall, $11 \%$ of our patients with pauci-immune GN were AA. Our study shows that AA patients with pauci-immune GN are younger at disease onset and are more often MPO-ANCA positive. There were no differences in treatment response (rates of complete remission or initial treatment resistance) between the groups. The rates of ESRD, relapse, and death in AA patients are similar to Caucasian patients.

Pauci-immune GN and AAV are rare in the AA population and variable in all ethnicities [8]. There is growing evidence that GPA mainly affects people of European background [11] and is rare in Japanese populations [12]. In contrast, MPA is seen with similar frequency to GPA in the Caucasian population but occurs at twofold or higher rates in the Japanese population [12]. Data from a multiracial population in France has indicated that AAV is more common among Europeans than among residents of northern or sub-Saharan African ancestry [13].

Previous reports on patients with pauci-immune GN have involved mostly Caucasian patients [1,14-18]. Previous reports from the GDCN cohort have documented that the total number of AA patients in their inception cohort of biopsy-proven vasculitis diagnosed between 1985 and 2009 was 58 (11\%) compared to 449 Caucasians [6]. In a recent study on ANCA-associated GN in an elderly population ( $>80$ years of age) out of New York, AA comprised only $4.1 \%$ of the patient population [19]. The European Vasculitis Study Group (EUVAS) vasculitis trials included $100 \%$ Caucasian patients $[14,16,20]$ while the AA 
patients enrolled in the 2trials conducted in the United States ranged from 1.1\% to 3\% of enrolled patients $[17,18]$. In contrast to these reports, AA patients in our study represented $11 \%$ of the entire cohort. This could be due to recent data that show differences in demographics and outcome of AAV patients enrolled in clinical trials compared to those followed in observational cohorts, with the trial patients being older, having worse renal disease, and experiencing higher early mortality within the first year [21].

The mean age in our cohort is similar to those reported in other cohorts $[6,15,18]$ and younger compared to patients enrolled in some European cohorts and trials [14,16,22,23]. The onset of disease occurred earlier in AA in our cohort compared to Caucasians and is similar to that in earlier reports from the GDCN [6]. Although both genders are usually equally affected by AAV, there has been a preponderance among females in some studies $[14,15]$ similar to what was observed among AA in our study.

The distribution of disease phenotypes was not significantly different between the 2 groups, although there was a slightly higher percent of patients with RLV and a lower percent of patients with MPA in AA compared to Caucasians. However, AA were more likely to be MPO-ANCA positive and this is in agreement with prior observations by the GDCN cohort [6]. The lungs and upper respiratory tract were the most frequently involved extra-renal organs among AA, and this was similar to Caucasians reported in other series [24]. The median eGFR at diagnosis was similar between AA and Caucasians. The eGFR at diagnosis is similar to that reported by Weidner et al. [25] and lower compared to what most vasculitis clinical trials have reported $[14,15,17,18]$. The percentage of patients requiring hemodialysis at presentation was $20 \%$ among AA. This is similar to those described among Caucasians in other studies [25,26] and lower than those described in other cohorts $[27,28]$.

The majority of our patients were treated with immunosuppressive therapy, and this is similar to other cohort studies $[29,30]$. AA patients were more likely to receive plasmapheresis. While this difference was likely due to a higher number of AA patients requiring hemodialysis at presentation, this difference was not adjusted for the usual indications of alveolar hemorrhage or dialysis-dependent renal failure for plasmapheresis in this retrospective study. In addition, our cohort included patients since 1983 and plasmapheresis was not routinely used in the management of AAV until the mid-1990s. Overall, $72 \%$ of our AA patients treated with immunosuppression went into remission compared to $82 \%$ of Caucasians. Similar rates of remission have been reported among Caucasians by other groups $[8,17,23,29,31]$ while higher remission rates are reported in some vasculitis trials $[14,15,18]$.

We anticipated higher treatment resistance in AA based on prior observations in AAV and lupus nephritis. Previous observations by the GDCN cohort report female gender, AA race, MPO-ANCA, and arteriosclerosis as predictors of treatment resistance [8]. In studies of lupus nephritis, AA patients have inferior response to immunosuppressive treatment and worse renal survival and patient survival compared to Caucasians [32]. Genetic factors leading to differences in disease severity are felt to play a role in AA due to differences in serologic and immunologic profiles among AA and Caucasians with lupus [32]. In our study, after controlling for age, gender, serum creatinine, and ANCA specificity, there was 
no difference in proportion of patients with initial treatment resistance. However, we were unable to look at histologic predictors in this retrospective study representing patients from 2 different institutions.

The rates of renal recovery in dialysis-dependent patients at presentation in our study are higher compared to those reported by other groups [8,33-36]. The mean eGFR at 12 and 24 months in the dialysis-independent patients is similar to those reported in the Cyclophosphamide Daily Oral Versus Pulsed (CYCLOPS) trial [20] and lower compared to other cohorts [34]. The relapse rates observed in AA in our study are similar to those reported by other cohorts $[8,23,25]$ but higher compared to those reported in some clinical trials $[14,20]$ and low compared to other series [37-39].

Overall, $16 \%$ of AA reached ESRD compared to $9 \%$ of Caucasians in our study. The rates of ESRD are similar to some studies [22] and lower compared to other reports [16,25]. In our study, $19 \%$ of AA died compared to $24 \%$ of Caucasians over a median follow-up time of 28 (IQR: 10, 57) months. These death rates were lower than reported in some clinical trials $[14,18,20]$ and similar compared to other cohorts [16,22,23,25,34].

Our study has certain limitations. The clinical outcomes were not correlated with histologic variables. Our study, like others, is limited by small patient numbers. Due to the high prevalence of hypertensive renal disease in AA, we can speculate that AAV may be underdiagnosed in AA. Despite differences in presentation, we are unable to show differences in response to treatment and outcomes. Nevertheless, our study provides new data for this rare but devastating condition in an AA cohort.

\section{Conclusion}

Pauci-immune glomerulonephritis is rare in AA patients. AA patients with vasculitis are younger at disease onset and more often MPO-ANCA positive compared to Caucasians. The potential benefit of the shorter time to diagnosis among AA did not result in better outcomes with multivariable analyses revealing no differences in treatment response and nearly equivalent rates of ESRD, renal relapse, and death rates by race over the entire time of follow-up.

\section{Acknowledgments}

Support and financial disclosure: Duvuru Geetha has served as a consultant for Genentech and received honraria for training sales force.

\section{References}

1. Booth AD, Almond MK, Burns A, Ellis P, Gaskin G, Neild GH, et al. Outcome of ANCAassociated renal vasculitis: a 5-year retrospective study. Am J Kidney Dis. 2003; 41:776-84. [PubMed: 12666064]

2. Falk RJ, Nachman PH, Hogan SL, Jennette JC. ANCA glomerulonephritis and vasculitis: a Chapel Hill perspective. Semin Nephrol. 2000; 20:233-43. [PubMed: 10855933]

3. Cohen BA, Clark WF. Pauci-immune renal vasculitis: natural history, prognostic factors, and impact of therapy. Am J Kidney Dis. 2000; 36:914-24. [PubMed: 11054347] 
4. Jennette JC, Falk RJ. Small-vessel vasculitis. N Engl J Med. 1997; 337:1512-23. [PubMed: 9366584]

5. Harris AA, Falk RJ, Jennette JC. Crescentic glomerulonephritis with a paucity of glomerular immunoglobulin localization. Am J Kidney Dis. 1998; 32:179-84. [PubMed: 9669443]

6. Cao Y, Schmitz JL, Yang J, Hogan SL, Bunch D, Hu Y, et al. DRB1*15 allele is a risk factor for PR3-ANCA disease in African Americans. J Am Soc Nephrol. 2011; 22:1161-7. [PubMed: 21617122]

7. Gelber AC, Manno RL, Shah AA, Woods A, Le EN, Boin F, et al. Race and association with disease manifestations and mortality in scleroderma: a 20-year experience at the Johns Hopkins Scleroderma Center and review of the literature. Medicine. 2013; 92:191-205. [PubMed: 23793108]

8. Hogan SL, Falk RJ, Chin H, Cai J, Jennette CE, Jennette JC, et al. Predictors of relapse and treatment resistance in antineutrophil cytoplasmic antibody-associated small-vessel vasculitis. Ann Intern Med. 2005; 143:621-31. [PubMed: 16263884]

9. Jennette JC, Falk RJ, Andrassy K, Bacon PA, Churg J, Gross WL, et al. Nomenclature of systemic vasculitides. Proposal of an international consensus conference. Arthritis Rheum. 1994; 37:187-92. [PubMed: 8129773]

10. Levey AS, Bosch JP, Lewis JB, Greene T, Rogers N, Roth D. A more accurate method to estimate glomerular filtration rate from serum creatinine: a new prediction equation. Modification of diet in Renal Disease Study Group. Ann Intern Med. 1999; 130:461-70. [PubMed: 10075613]

11. Gibelin A, Maldini C, Mahr A. Epidemiology and etiology of wegener granulomatosis, microscopic polyangiitis, churg-strauss syndrome and goodpasture syndrome: vasculitides with frequent lung involvement. Semin Respir Crit Care Med. 2011; 32:264-73. [PubMed: 21674413]

12. Fujimoto S, Watts RA, Kobayashi S, Suzuki K, Jayne DR, Scott DG, et al. Comparison of the epidemiology of anti-neutrophil cytoplasmic antibody-associated vasculitis between Japan and the U. K Rheumatology (Oxford). 2011; 50:1916-20.

13. Mahr A, Guillevin L, Poissonnet M, Ayme S. Prevalences of polyarteritis nodosa, microscopic polyangiitis, Wegener's granulomatosis, and Churg-Strauss syndrome in a French urban multiethnic population in 2000: a capture-recapture estimate. Arthritis Rheum. 2004; 51:92-9. [PubMed: 14872461]

14. Jayne D, Rasmussen N, Andrassy K, Bacon P, Tervaert JW, Dadoniene J, et al. A randomized trial of maintenance therapy for vasculitis associated with antineutrophil cytoplasmic autoantibodies. N Engl J Med. 2003; 349:36-44. [PubMed: 12840090]

15. De Groot K, Rasmussen N, Bacon PA, Tervaert JW, Feighery C, Gregorini G, et al. Randomized trial of cyclophosphamide versus methotrexate for induction of remission in early systemic antineutrophil cytoplasmic antibody-associated vasculitis. Arthritis Rheum. 2005; 52:2461-9. [PubMed: 16052573]

16. Jayne DR, Gaskin G, Rasmussen N, Abramowicz D, Ferrario F, Guillevin L, et al. Randomized trial of plasma exchange or high-dosage methylprednisolone as adjunctive therapy for severe renal vasculitis. J Am Soc Nephrol. 2007; 18:2180-8. [PubMed: 17582159]

17. Stone JH, Merkel PA, Spiera R, Seo P, Langford CA, Hoffman GS, et al. Rituximab versus cyclophosphamide for ANCA-associated vasculitis. N Engl J Med. 2010; 363:221-32. [PubMed: 20647199]

18. Wegener's Granulomatosis Etanercept Trial (WGET) Research Group. Etanercept plus standard therapy for Wegener's granulomatosis. N Engl J Med. 2005; 352:351-61. [PubMed: 15673801]

19. Bomback AS, Appel GB, Radhakrishnan J, Shirazian S, Herlitz LC, Stokes B, et al. ANCAassociated glomerulonephritis in the very elderly. Kidney Int. 2011; 79:757-64. [PubMed: 21160463]

20. de Groot K, Harper L, Jayne DR, Flores Suarez LF, Gregorini G, Gross WL, et al. Pulse versus daily oral cyclophosphamide for induction of remission in antineutrophil cytoplasmic antibodyassociated vasculitis: a randomized trial. Ann Intern Med. 2009; 150:670-80. [PubMed: 19451574]

21. Pagnoux C, Carette S, Khalidi NA, Cuthberston C, Fortin P, Hoffman G, et al. Are patients with ANCAAAV entered into clinical trials representative of patients followed in observational cohorts? Clin Exp Immunol. 2011; 164:81-2. 
22. Day CJ, Howie AJ, Nightingale P, Shabir S, Adu D, Savage CO, et al. Prediction of ESRD in pauci-immune necrotizing glomerulonephritis: quantitative histomorphometric assessment and serum creatinine. Am J Kidney Dis. 2010; 55:250-8. [PubMed: 20045237]

23. Smith RM, Jones RB, Guerry MJ, Laurino S, Catapano F, Chaudhry A, et al. Rituximab for remission maintenance in relapsing antineutrophil cytoplasmic antibody-associated vasculitis. Arthritis Rheum. 2012; 64:3760-9. [PubMed: 22729997]

24. Hedger N, Stevens J, Drey N, Walker S, Roderick P. Incidence and outcome of pauci-immune rapidly progressive glomerulonephritis in Wessex, UK: a 10-year retrospective study. Nephrol Dial Transplant. 2000; 15:1593-9. [PubMed: 11007827]

25. Weidner S, Geuss S, Hafezi-Rachti S, Wonka A, Rupprecht HD. ANCA-associated vasculitis with renal involvement: an outcome analysis. Nephrol Dial Transplant. 2004; 19:1403-11. [PubMed: 15069175]

26. Lionaki S, Hogan SL, Jennette CE, Hu Y, Hamra JB, Jennette JC, et al. The clinical course of ANCA small-vessel vasculitis on chronic dialysis. Kidney Int. 2009; 76:644-51. [PubMed: 19536079]

27. Rihova Z, Jancova E, Merta M, Rysava R, Reiterova J, Zabka J, et al. Long-term outcome of patients with antineutrophil cytoplasmic autoantibody-associated vasculitis with renal involvement. Kidney Blood Press Res. 2005; 28:144-52. [PubMed: 15908752]

28. Neumann I, Kain R, Regele H, Soleiman A, Kandutsch S, Meisl FT. Histological and clinical predictors of early and late renal outcome in ANCA-associated vasculitis. Nephrol Dial Transplant. 2005; 20:96-104. [PubMed: 15546891]

29. Chen M, Yu F, Wang SX, Zou WZ, Zhao MH, Wang HY. Antineutrophil cytoplasmic autoantibody-negative pauci-immune crescentic glomerulonephritis. J Am Soc Nephrol. 2007; 18:599-605. [PubMed: 17215440]

30. Eisenberger U, Fakhouri F, Vanhille P, Beaufils H, Mahr A, Guillevin L, et al. ANCA-negative pauci-immune renal vasculitis: histology and outcome. Nephrol Dial Transplant. 2005; 20:1392-9. [PubMed: 15855209]

31. Hoffman GS, Kerr GS, Leavitt RY, Hallahan CW, Lebovics RS, Travis WD, et al. Wegener granulomatosis: an analysis of 158 patients. Ann Intern Med. 1992; 116:488-98. [PubMed: 1739240]

32. Korbet SM, Evans J, Lewis EJ. Collaborative Study Group. Severe lupus nephritis: racial differences in presentation and outcome. J Am Soc Nephrol. 2007; 18:244-54. [PubMed: 17167111]

33. de Lind van Wijngaarden RA, Hauer HA, Wolterbeek R, Jayne DR, Gaskin G, Rasmussen N, et al. Chances of renal recovery for dialysis-dependent ANCA-associated glomerulonephritis. J Am Soc Nephrol. 2007; 18:2189-97. [PubMed: 17596637]

34. Slot MC, Tervaert JW, Franssen CF, Stegeman CA. Renal survival and prognostic factors in patients with PR3-ANCA associated vasculitis with renal involvement. Kidney Int. 2003; 63:670 7. [PubMed: 12631133]

35. Nachman PH, Hogan SL, Jennette JC, Falk RJ. Treatment response and relapse in antineutrophil cytoplasmic autoantibody-associated microscopic polyangiitis and glomerulonephritis. J Am Soc Nephrol. 1996; 7:33-9. [PubMed: 8808107]

36. Mekhail TM, Hoffman GS. Longterm outcome of Wegener's granulomatosis in patients with renal disease requiring dialysis. J Rheumatol. 2000; 27:1237-40. [PubMed: 10813293]

37. Sanders JS, Huitma MG, Kallenberg CG, Stegeman CA. Prediction of relapses in PR3-ANCAassociated vasculitis by assessing responses of ANCA titres to treatment. Rheumatology (Oxford). 2006; 45:724-9. [PubMed: 16399845]

38. Hiemstra TF, Walsh M, Mahr A, Savage CO, de Groot K, Harper L, et al. Mycophenolate mofetil vs azathioprine for remission maintenance in anti-neutrophil cytoplasmic antibody-associated vasculitis: a randomized controlled trial. J Am Med Assoc. 2010; 304:2381-2388.

39. Pagnoux C, Hogan SL, Chin H, Jennette JC, Falk RJ, Guillevin L, et al. Predictors of treatment resistance and relapse in antineutrophil cytoplasmic antibody-associated small-vessel vasculitis: comparison of two independent cohorts. Arthritis Rheum. 2008; 58:2908-18. [PubMed: 18759282] 


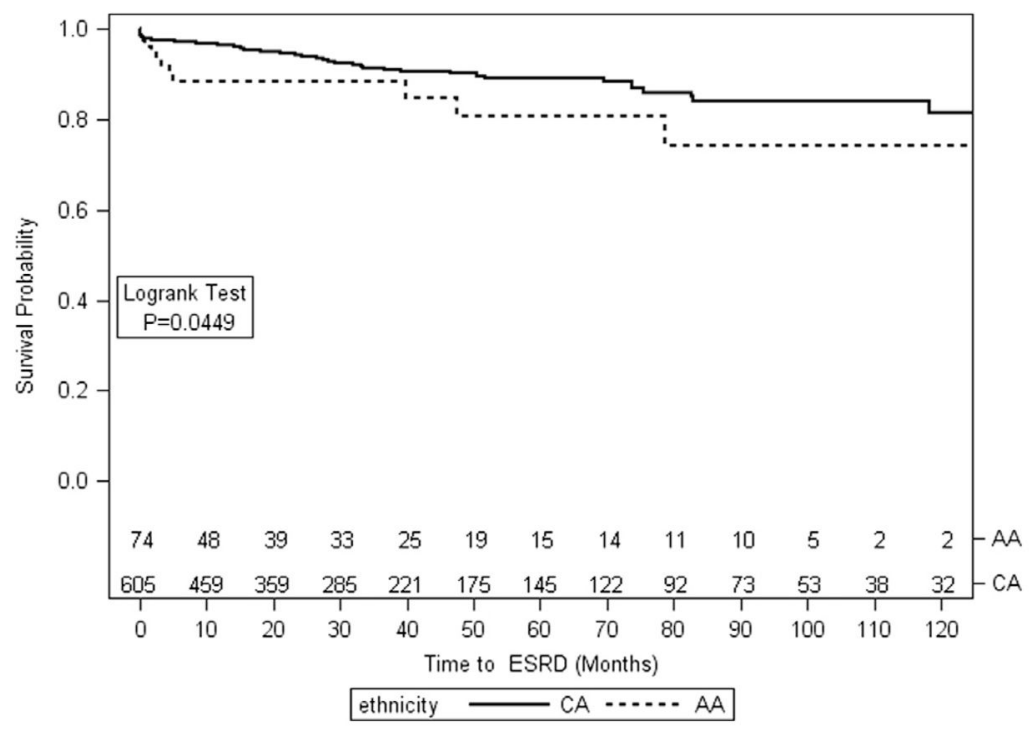

Fig.

Kaplan-Meier curve of kidney survival. 
Table 1

Comparison of demographics, disease phenotype, and induction therapy

\begin{tabular}{|c|c|c|c|}
\hline & Caucasian $(N=597,89 \%)$ & $\mathrm{AA}(N=75,11 \%)$ & $p$-Value ${ }^{a}$ \\
\hline Age (years) at diagnosis, mean \pm SD & $57 \pm 18$ & $52 \pm 20$ & 0.051 \\
\hline Females & $268(45 \%)$ & $40(53 \%)$ & 0.18 \\
\hline ANCA type ${ }^{b}$ & & & 0.0114 \\
\hline Both & $2(0.34 \%)$ & $0(0 \%)$ & \\
\hline $\mathrm{PR} 3 / \mathrm{c}-\mathrm{ANCA}$ & $260(44 \%)$ & $19(25 \%)$ & \\
\hline $\mathrm{MPO} / \mathrm{p}-\mathrm{ANCA}$ & $321(54 \%)$ & $53(71 \%)$ & \\
\hline ANCA neg. & $13(2 \%)$ & $3(4 \%)$ & \\
\hline Vasculitis type & & & 0.17 \\
\hline EGPA & $2(0.34 \%)$ & $1(1 \%)$ & \\
\hline GPA & $153(26 \%)$ & $19(25 \%)$ & \\
\hline Renal limited & $122(20 \%)$ & $21(28 \%)$ & \\
\hline MPA & $320(54 \%)$ & $34(45 \%)$ & \\
\hline \multicolumn{4}{|l|}{ eGFR at diagnosis (ml/min) } \\
\hline Median (IQR) & $21(12,38)$ & $22(14,45)$ & 0.54 \\
\hline \multicolumn{4}{|c|}{ Time from symptoms to renal biopsy (months) } \\
\hline Median (IQR) & $0.66(0.00,3.62)$ & $0.23(0.00,1.22)$ & 0.0028 \\
\hline \multicolumn{4}{|l|}{ Organs } \\
\hline Lung $b$ & $307(52 \%)$ & $41(55 \%)$ & 0.63 \\
\hline Upper respiratory $b$ & $221(37 \%)$ & $22(29 \%)$ & 0.20 \\
\hline Kidney & $597(100 \%)$ & $75(100 \%)$ & \\
\hline Induction with plasmapheresis $b$ & $78(13 \%)$ & $20(27 \%)$ & 0.0047 \\
\hline Induction therapy & & & 0.48 \\
\hline Prednisone alone & $54(9 \%)$ & $8(11 \%)$ & \\
\hline Cyclophosphamide & $486(81 \%)$ & $60(80 \%)$ & \\
\hline Others $^{c}$ & $40(7 \%)$ & $3(4 \%)$ & \\
\hline Not treated & $17(3 \%)$ & $4(5 \%)$ & \\
\hline Follow-up (months), median (IQR) & $26(10,55)$ & $28(5,52)$ & 0.64 \\
\hline
\end{tabular}

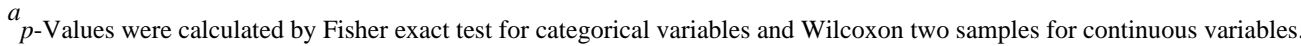

$b_{\text {There was }} 1$ patient who had missing data for ANCA type and 1 patient who had missing data for lung involvement, 2 patients who had missing data for upper respiratory tract involvement and 2 patients who had missing data for induction therapy.

${ }^{c}$ Azathioprine, mycophenolate mofetil, methotrexate, rituximab, and intravenous immunoglobulin. 
Table 2

Outcomes by race: remission, relapse, ESRD, and death

\begin{tabular}{|c|c|c|c|}
\hline Outcome parameters & Caucasian $(N=597)$ & $\mathbf{A A}(N=75)$ & $p$-Values $a$ \\
\hline Treatment response ${ }^{b}$ & $n=563$ & $n=69$ & 0.0504 \\
\hline Remission & $464(82 \%)$ & $50(72 \%)$ & \\
\hline Resistance & $99(18 \%)$ & $19(28 \%)$ & \\
\hline Acute dialysis at diagnosis & $78(13 \%)$ & $14(19 \%)$ & 0.21 \\
\hline ESKD ever & $53(9 \%)$ & $12(16 \%)$ & 0.06 \\
\hline Early ESKD (<3 months) & $18(3 \%)$ & $6(8 \%)$ & 0.0412 \\
\hline Median months to ESKD (IQR) & $16(0.3,33)$ & $3(0.4,22)$ & 0.29 \\
\hline \multirow[t]{3}{*}{ eGFR at 6 months $(\mathrm{ml} / \mathrm{min})(n$, mean $\pm \mathrm{SD}$, median, and $\mathrm{IQR})$} & $303 / 597$ & $22 / 75$ & 0.55 \\
\hline & $38 \pm 22$ & $44 \pm 30$ & \\
\hline & $34(22,49)$ & $39(20,70)$ & \\
\hline \multirow[t]{3}{*}{ eGFR at 1 year $(\mathrm{ml} / \mathrm{min})(n$, mean $\pm \mathrm{SD}$, median, and IQR $)$} & $261 / 597$ & $23 / 75$ & 0.48 \\
\hline & $40 \pm 23$ & $48 \pm 36$ & \\
\hline & $35(23,54)$ & $41(21,72)$ & \\
\hline \multirow[t]{3}{*}{ eGFR at 2 years $(\mathrm{ml} / \mathrm{min})(n$, mean $\pm \mathrm{SD}$, median, and $\mathrm{IQR})$} & $195 / 597$ & $14 / 95$ & 0.0786 \\
\hline & $40 \pm 24$ & $51 \pm 25$ & \\
\hline & $35(23,53)$ & $45(35,71)$ & \\
\hline Renal relapse $n(\%)^{c}$ & $n=464$ & $n=50$ & \\
\hline \multirow[t]{2}{*}{ Median months to relapse (IQR) } & $137(30 \%)$ & $12(24 \%)$ & 0.51 \\
\hline & $18(9,38)$ & $19(8,36)$ & 0.85 \\
\hline Deaths, $n(\%)$ & $144(24 \%)$ & $14(19 \%)$ & 0.39 \\
\hline Median months to death (IQR) & $31(6,65)$ & $36(17,64)$ & 0.84 \\
\hline
\end{tabular}

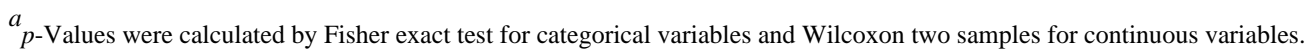

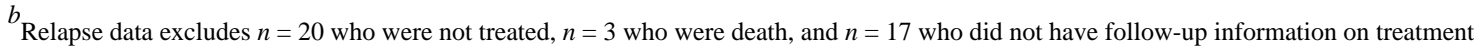
response.

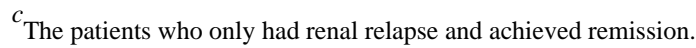



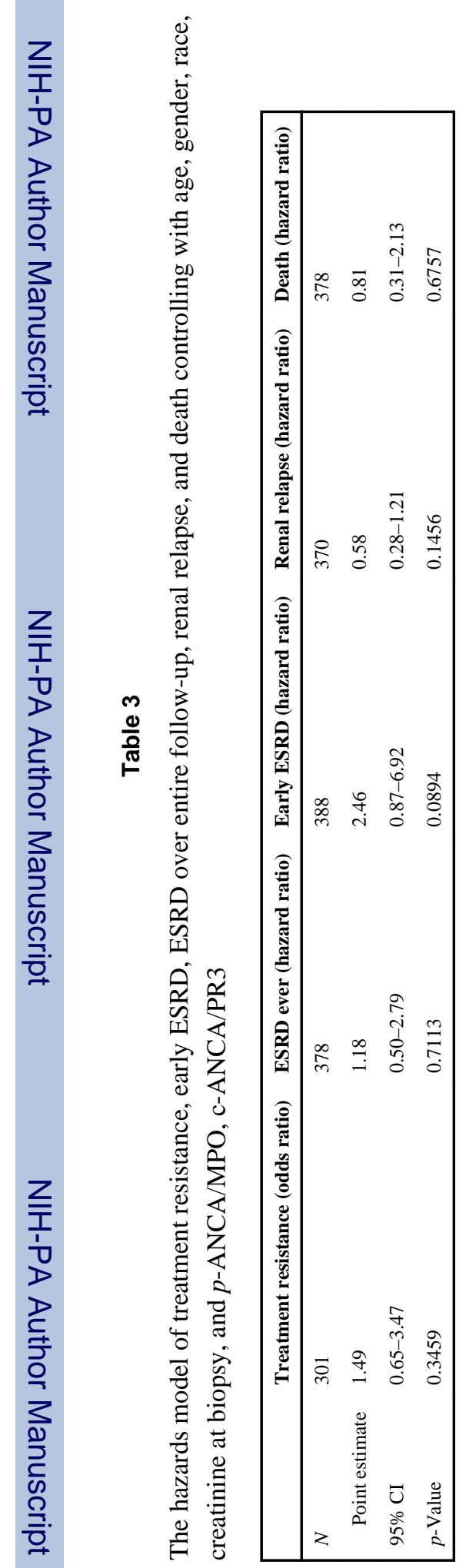

Semin Arthritis Rheum. Author manuscript; available in PMC 2014 July 08. 